A) Check for updates

Cite this: Food Funct., 2020, 11 10022

Received 13th July 2020, Accepted 22nd October 2020

DOI: 10.1039/dOfo01835j

rsc.li/food-function

\section{Differences in oral processing behavior of consumers varying in age, gender and ethnicity lead to changes in bolus properties but only to small differences in dynamic texture perception of sausages $\dagger$}

\author{
Monica G. Aguayo-Mendoza, (D) *a,b Eduardo F. Martinez-Almaguer, ${ }^{b}$ \\ Betina Piqueras-Fiszman ${ }^{c}$ and Markus Stieger ${ }^{a, d}$
}

\begin{abstract}
Consumer characteristics such as age, gender and ethnicity influence food oral processing behavior. The aim of this study was to determine the effect of age, gender and ethnicity on consumption time, bolus properties and dynamic sensory perception of sausages. Consumption time, bolus properties (saliva incorporation, particle size distribution and rheological properties) and dynamic texture perception (Temporal Dominance of Sensations, TDS) of sausages were compared between young Dutch, Caucasians ( $n=21 ; 22 \pm 2.8$ years), young Chinese, Asians ( $n=21 ; 23 \pm 1.6$ years), and elderly Dutch, Caucasians ( $n=22 ; 70 \pm 4.3$ years). Elderly Dutch masticated the sausage $22 \%$ longer $(25.6 \mathrm{~s}$ ) than young Dutch consumers $(21.0 \mathrm{~s})$. Elderly Dutch produced sausage boli that were softer, more adhesive, less cohesive and contained more particles than those of young Dutch adults. Elderly females produced more adhesive and less cohesive sausage bolus than males. Young Chinese females had $75.6 \%$ longer consumption time $(29.5 \mathrm{~s}$ ) than young Dutch females (16.8 s). Young Chinese males masticated the sausages in less time (18.8 s) than young Chinese females (29.5 s). Young Chinese produced softer and less cohesive bolus with slightly smaller and more particles than young Dutch. Saliva incorporation and bolus particle size were not affected by age, gender and ethnicity. Mediation analysis revealed that the effect of consumer characteristics such as age, gender and ethnicity on bolus properties was mediated by consumption time. At the beginning and end of consumption time, dynamic texture perception of sausages was similar for all consumer groups and strongly correlated with bolus properties. Differences in dynamic texture perception between consumer groups were observed only during the middle stages of mastication with low dominance rates. We conclude that consumers differing in age, gender and ethnicity vary in oral processing time to produce bolus with textural properties optimized to their needs. Furthermore, consumption time is the underlying mechanism that explains the differences in bolus properties between the consumer groups. While variations in consumption time of sausages lead to considerable differences in bolus properties, it only leads to small differences in dynamic texture perception.
\end{abstract}

\section{Introduction}

In order to form a swallowable bolus, oral processing behavior is adapted to the rheological and mechanical properties of foods by modifying bite size, consumption time, number of chews $^{1,2}$ and/or muscle activity. ${ }^{3}$ Food oral processing behavior also depends on consumer characteristics such as age, gender and ethnicity. Several studies demonstrated that elderly masticate foods with more chewing cycles, longer chewing times and lower eating rates than young consumers. ${ }^{4-7}$ Females chew foods longer than males and take smaller bites leading to lower eating rates than males. ${ }^{5,7-9}$ While the influence of age 
and gender on food oral processing behavior has been studied extensively, little is known about the effect of ethnicity on food oral processing behavior. Recent studies indicated that bite size and eating rate are lower in Asians than Caucasians. ${ }^{5}$ Differences in oral processing behavior may impact the structural transitions of the bolus during mastication and in turn might impact sensory perception. ${ }^{10}$

The goal of oral processing is to achieve a ready and safe to swallow bolus. ${ }^{11,12}$ Solid foods are broken down into many small particles during oral processing and lubricated with saliva and serum released from the food. ${ }^{13}$ The swallowing threshold depends mainly on the level of structure breakdown and the lubrication properties of the bolus. Bolus formation and properties may be impacted by oral processing behavior, so that differences in oral processing behavior contribute to differences in bolus properties. How bolus properties evolve during mastication and when the bolus is sensed as safe to swallow may depend on individual characteristics of the consumer and may vary between groups of consumers.

During bolus formation in the oral cavity the sensory properties of the food are constantly evaluated. Dynamic perception of texture attributes may be linked to dynamic structural transformations of the bolus. Differences in sensory perception upon changes in oral behavior have been reported previously for various foods. ${ }^{14,15}$ Currently, there is a lack of understanding how differences in oral processing behaviour between consumer groups lead to differences in bolus properties and consequently differences in sensory perception. Such knowledge could provide insights to tailor foods towards specific consumer groups based on consumer characteristics. The aim of this study was to determine the influence of age, gender and ethnicity on consumption time, bolus properties and dynamic texture perception of sausages. Consumption time was chosen as the variable to quantify oral processing behavior based on previous findings that show that consumption time and bite size are the most relevant parameters for oral processing. ${ }^{1,5}$ Bolus properties (saliva incorporation, particle size distribution and rheological properties) were analyzed and compared between consumer groups varying in age, gender and ethnicity. Temporal dominance of sensations (TDS) was used to compare dynamic texture perception of sausages between consumer groups and to link bolus properties to dynamic sensory perception.

\section{Materials and methods}

\subsection{Subjects}

Sixty-four subjects were recruited through social media and printed advertisements: $n=21$ young Dutch, Caucasians (eleven females and ten males, $22 \pm 2.8$ years); $n=21$ young Chinese, Asians (twelve female and nine males, $23 \pm 1.6$ years) living in The Netherlands and outside China for less than one year; and $n=22$ elderly Dutch, Caucasians (eleven females and eleven males, $70 \pm 4.3$ years). All participants were healthy (self-reported), without swallowing or mastication disorders and had good dental status with no missing teeth (except for third molars or wisdom teeth). Prior to the study, potential participants attended an information session and received a written leaflet. After personal deliberation, subjects signed an informed consent. Upon completion of the study all participants were reimbursed. The study was conducted in line with the Declaration of Helsinki.

\subsection{Test food and serving size}

Commercially available canned hotdog sausages (Unox® Hotdogs, Unilever, The Netherlands) were used. For bolus collection and sensory evaluations, hotdog sausages were cut into cylinders of $20 \mathrm{~mm}$ diameter and $30 \mathrm{~mm}$ height with a constant weight of $7 \mathrm{~g}$ and served at room temperature without further preparation.

\subsection{Consumption time}

Consumption time was measured per subject in triplicate to determine consumption time corresponding to $100 \%$ of mastication of one sample. Participants were asked to chew it normally and to indicate the main swallowing moment by raising a hand. The time needed to consume each sausage sample was recorded by the researcher and the average time of three replicates per participant determined. This average was considered $100 \%$ of mastication time of the participant.

\subsection{Bolus collection and characterization}

Collection of sausage boli was done in two sessions of $30 \mathrm{~min}$ each. During each session, participants were instructed to place the sausage sample $(7 \mathrm{~g})$ in their mouth, chew it as they would normally do for their individual 33, 66 or $100 \%$ of mastication time, and expectorate the bolus in a coded container. To provide participants with a comfortable atmosphere, bolus collection sessions were held individually. The expectorated sausage boli collected in the first session were used to determine particle size distribution and saliva incorporation. Expectorated boli collected in the second session were used to quantify rheological bolus properties.

2.4.1 Particle number and size analysis. Participants expectorated the sausage bolus directly into a Petri dish, then a glycerol-water $(1: 2)$ solution was added to the bolus allowing the separation of bolus particles. To improve separation of particles further, they were gently manually separated with a spatula. Petri dishes were placed on a scanner (CanonScan $9000 \mathrm{~F}$ markII) and images obtained in greyscale ( 8 bit) with a resolution of $1200 \mathrm{dpi}$. Images were analyzed using ImageJ (National Institutes of Health, Version $1.51 \mathrm{~g}$ ) and the median particle size in $\mu \mathrm{m}\left(d_{50}\right)$ and number of particles determined. This procedure was done in duplicate per participant for each expectoration time point (33, 66 and 100\% mastication time).

2.4.2 Moisture content and bolus saliva incorporation. Boli were expectorated into aluminium plates and wet bolus weight was determined. Boli were dried for $16 \mathrm{~h}$ at $105{ }^{\circ} \mathrm{C}$ in an atmospheric oven (Venti-line line, VWR®). After drying, samples were weighed again. This procedure was done in duplicate per participant and for each expectoration time 
point (33, 66 and 100\% mastication time). Saliva content was calculated following the method described by Drago et al. ${ }^{16}$ which takes into account the amount of food that remains in the mouth after oral processing. The mass ratio of saliva incorporated into the bolus (mg) per gram of food masticated was calculated $\left(h_{\mathrm{w}}\right)$ as follows:

$$
h_{\mathrm{w}}=\frac{\mathrm{DM}_{\text {sample }} \times 1000}{\mathrm{DM}_{\text {spit_bolus }}}-1000 .
$$

2.4.3 Mechanical bolus properties determined by two cycle penetration tests. To obtain boli with cylindrical shapes of similar dimensions, participants expectorated bolus into a $35 \mathrm{~mm}$ diameter cup with a line marked at $25 \mathrm{~mm}$ height. Boli surfaces were gently evened with the flat side of the plunger of a $50 \mathrm{~mL}$ syringe. Immediately after expectoration, boli were analyzed by a two-cycle penetration test using a Texture Analyzer (TA. XT plus) equipped with a load cell of $5 \mathrm{~kg}$ and a cylindrical steel probe of $20 \mathrm{~mm}$ diameter. Two successive penetration cycles were performed at a constant speed of $5 \mathrm{~mm} \mathrm{~s}^{-1}$ up to $50 \%$ strain with a resting time of $5 \mathrm{~s}$ between both penetration cycles. Three replicates were performed for all boli expectorated after 33, 66 and $100 \%$ of mastication time of each participant. From the two cycle penetration tests, maximum force $(\mathrm{N})$ of first penetration (firmness), negative area under force-time curve $(\mathrm{N} \mathrm{s})$ between the moment that the probe is retrieved after first penetration until second penetration (adhesiveness) and ratio between area under the force-time curve of the second and first penetration cycle (cohesiveness) were extracted.

\subsection{Sensory evaluation}

2.5.1 Texture attribute generation. A preliminary list of 13 attributes describing texture perception of sausages was obtained from literature. ${ }^{14,17-24}$ To generate the list of attributes used for Temporal Dominance of Sensation (TDS), these 13 attributes were evaluated by $n=43$ subjects from the consumer groups using a Check-All-That-Apply evaluation of the sausage. The eight most frequently checked texture attributes (Table 1) were selected to be used in the TDS evaluation.

2.5.2 TDS evaluation. A $60 \mathrm{~min}$ familiarization session was performed to introduce the concept of temporality of sensations and to explain the dominant sensation as "the attribute associated with the sensation catching the attention at a given time, and not necessarily the attribute with the highest intensity". ${ }^{25}$ Afterwards, texture attributes were presented to the subjects together with their definitions. In addition, sub- jects performed a TDS test with a dummy sausage sample to familiarize themselves with the sensory methodology and software used (EyeQuestion v3.9.7). Subjects were encouraged to ask questions at any time during this familiarization session. No further training was done in order to avoid biasing the oral processing behavior of subjects. ${ }^{26}$

Sensory evaluation was performed in a well-lit room with a portable separator used to create sensory booths. During one session of $30 \mathrm{~min}$, participants $(n=21$ young Dutch, Caucasians; $n=21$ young Chinese, Asians; $n=22$ elderly Dutch, Caucasians) received a dummy sausage sample and the hotdog sausage sample in triplicate (6 samples per session per subject). A dummy sample was included for randomization and blinding purposes since we were interested only in the texture evaluation of one type of sausage. Hotdog sausage samples were presented one by one on a plate labeled with three-digit codes following a counterbalanced design. Participants were instructed to put the sausage sample $(7 \mathrm{~g})$ into their mouth and click the start button to begin the evaluation. The texture attributes were presented simultaneously on a computer screen. Subjects were free to select an attribute several times or to not select it at all throughout the evaluation. To facilitate the assessment and to avoid confusion, attributes were presented in random order across the panel but remained in the same position for a given panelist during the whole session. Finally, participants pressed the stop button to indicate the swallowing moment. Between samples participants were asked to neutralize their palate by eating an unsalted cracker and rinsing their mouth with water.

\subsection{Data analysis}

A two-way ANOVA was performed to determine the effect of gender and age and their interaction on consumption time and bolus properties at the swallowing point (100\% mastication time). Another two-way ANOVA was performed to determine the effect of gender and ethnicity and their interaction on consumption time and bolus properties at the swallowing point (100\% mastication time). Effects were considered statistically significant at $p<0.05$. When applicable, post-hoc analyses were performed with Bonferroni adjustment.

Mediation analyses were performed to determine the effect of age, gender and ethnicity on bolus properties (saliva incorporation, median particle size, particle number, hardness, cohesiveness, adhesiveness) using consumption time as mediator (Fig. 1).

Table 1 Texture attributes and definitions used for TDS evaluation of hotdog sausages

\begin{tabular}{|c|c|}
\hline Attribute & Definition \\
\hline Firmness & Force needed to bite through the sausage \\
\hline Chewiness & Amount of work required to chew the sausage into a state that is ready to swallow \\
\hline Juiciness & Water release from sausage perceived during mastication \\
\hline Graininess & From crumbly to granular particles in the mouth \\
\hline Smoothness & Homogeneity of the sausage in the mouth \\
\hline Fattiness & Perception of fat \\
\hline Slippery & Easiness to move the sausage in the mouth \\
\hline Dryness & Rough feeling in the mouth \\
\hline
\end{tabular}




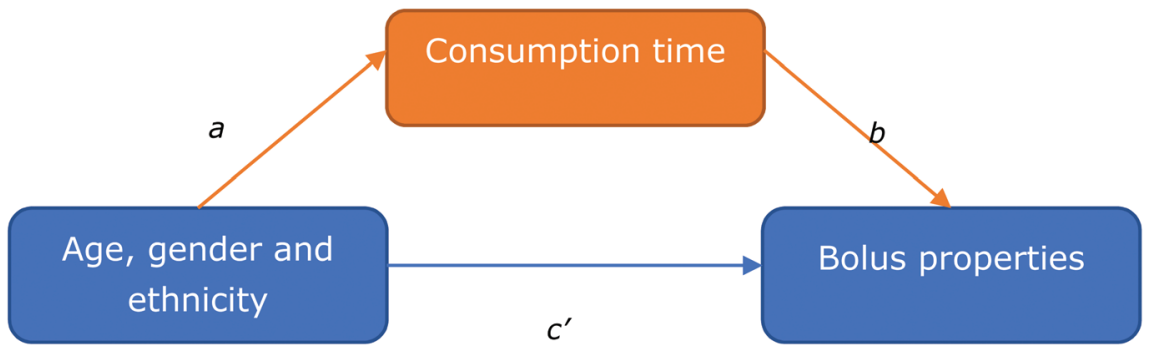

Fig. 1 Mediation model of age, gender and ethnicity as predictors for the outcome variables of bolus properties, mediated by the consumption time. The regression coefficients $a, b$, and $c^{\prime}$ estimate the effect of the predictor on the mediator, the effect of the mediator on the outcome variable, and the direct effect of the predictor on the outcome variable, respectively.

The effect of age and gender on bolus properties was determined between the groups of young Dutch $(n=21)$ and elderly Dutch $(n=22)$. To determine the effect of ethnicity on bolus properties, young Dutch $(n=21)$ were compared to young Chinese $(n=21)$. Indirect effects were estimated by bootstrapping using 5000 bootstrap samples and reported with bias corrected confidence intervals. When the confidence intervals did not include zero, mediation was considered existent. Data are presented as mean \pm standard error of the mean (SEM).

To illustrate the evolution of sensory perception, TDS curves were built by plotting dominance rate (proportion of subjects who scored each attribute at each point of time as dominant sensation) against consumption time. Data used to build the curves were time standardized on a scale from 0 (starting point) to 100 (swallowing moment). Chance and significance level were calculated as described by Pineau. ${ }^{25}$

To investigate the relationships between bolus properties at three expectoration times and dynamic texture perception from TDS, a Multiple Factor Analysis (MFA) was performed with two data matrices as active datasets. Bolus properties collected at the different expectoration time points $(33,66$ and $100 \%$ mastication time; see Appendix A.1) formed one data matrix. The second matrix included the values of the cumulative dominance rate for each attribute at during three time periods 33 (T0-T33), 66 (T34-T66) and 100 (T67-T100) of standardized consumption time. Cumulative dominance rate was calculated by summing the significant dominance rates of each attribute per period. Individuals' graphs and correlations circles were plotted for dimensions one and two.

Data analyses were performed in R v3.6.3. Two-way ANOVA analyses were performed using rstatix package v0.4.0. TDS curves and sensory trajectories were constructed using tempR package v0.9.9.16. MFA analysis were performed with packages FactoMineR v2.3 and factoextra v1.0.7. Mediation analysis was performed using SPSS v24.0 and the PROCESS macro v3.00.

\section{Results}

\subsection{Effect of age, gender and ethnicity on consumption time}

Age had a significant main effect $[F(1,125)=27.37, p<0.001]$ on consumption time among Dutch consumers, with elderly having longer consumption time (25.6 $\pm 1.4 \mathrm{~s})$ than young adults $(21.0 \pm$ 0.9 s) (Table 2). Gender significantly interacted with ethnicity affecting consumption time $[F(1,122)=15.21, p<0.001]$. Chinese males $(18.8 \pm 1.1 \mathrm{~s})$ had a shorter consumption time than Chinese females $(29.5 \pm 1.8 \mathrm{~s})[F(1,122)=28.2, p<0.001]$, and Chinese females had longer consumption time $(29.5 \pm 1.8 \mathrm{~s})$ than Dutch females $(16.8 \pm 1.1 \mathrm{~s})[F(1,122)=44.4, p<0.001]$ (Table 2).

\subsection{Effect of age, gender and ethnicity on bolus properties at the swallowing point}

Table 2 shows descriptive data of all consumer groups for the measured bolus properties at swallowing point. Age had a significant main effect on bolus hardness $[F(1,125)=5.79, p=0.018]$ among Dutch consumers with elderly producing softer bolus $(1.95 \mathrm{~N})$ than young adults $(2.30 \mathrm{~N})$. Age significantly interacted with gender affecting bolus adhesiveness $[F(1,122)=6.09, p=$ $0.015]$, cohesiveness $[F(1,125)=4.32, p=0.040]$, and number of bolus particles $[F(1,82)=4.27, p=0.042]$. It was shown that elderly Dutch females produced a more adhesive $(0.05 \mathrm{~N} \mathrm{~s})$ and less cohesive (0.37) bolus containing more particles (561) than young Dutch females ( $0.03 \mathrm{~N} \mathrm{~s}, 0.41$ and 381, respectively). Similarly, elderly Dutch females produced more adhesive $(0.05 \mathrm{~N} \mathrm{~s})$ and less cohesive (0.37) bolus than elderly Dutch males $(0.03 \mathrm{~N} \mathrm{~s}$ and 0.39 , respectively). Young Dutch females produced fewer particles (381) than young Dutch males (525). Age and gender did not have a significant effect on saliva ratio $h_{\mathrm{w}}$ and bolus particle size $d_{50}$.

Ethnicity had a significant main effect on bolus hardness $[F(1,125)=6.79, p=0.010]$, bolus cohesiveness $[F(1,122)=9.93$, $p=0.002]$, and particle size $d_{50}[F(1,80)=3.99, p=0.049]$. Chinese consumers produced softer $(1.95 \mathrm{~N})$, less cohesive $(0.38)$ bolus with slightly smaller particles $(1940 \mu \mathrm{m})$ than Dutch $(2.31 \mathrm{~N}, 0.41,1988 \mu \mathrm{m})$. Ethnicity significantly interacted with gender on number of particles $[F(1,80)=10.17, p<$ $0.001]$. Chinese females produced bolus with more particles (623) than Dutch females (381). Ethnicity did not have a significant effect on bolus adhesiveness and saliva incorporation.

\subsection{Mediation effect of consumption time on bolus properties of consumers varying in age, gender and ethnicity}

Table 3 shows the mediation coefficients that represent the effects of age, gender and ethnicity on the different bolus properties at the swallowing point. Age indirectly influenced saliva 
Table 2 Descriptive data (mean \pm SE) of consumption time and bolus properties of consumers differing in age ( $n=22$ elderly Dutch, $n=21$ young Dutch), ethnicity ( $n=21$ young Dutch, $n=21$ young Chinese), and gender ( $n=34$ females, $n=30$ males). Bolus data was collected at the swallowing point $(100 \%$ mastication time)

\begin{tabular}{|c|c|c|c|c|c|c|}
\hline & \multicolumn{4}{|l|}{ Young adults } & \multicolumn{2}{|l|}{ Elderly } \\
\hline & \multicolumn{2}{|l|}{ Chinese } & \multicolumn{4}{|l|}{ Dutch } \\
\hline & Females & Males & Females & Males & Females & Males \\
\hline Total consumption time (s) & $29.5 \pm 1.8$ & $18.8 \pm 1.1$ & $16.8 \pm 1.1$ & $17.2 \pm 1.4$ & $27.6 \pm 2.3$ & $23.5 \pm 1.5$ \\
\hline Hardness $(\mathrm{N})$ & $1.79 \pm 0.1$ & $2.12 \pm 0.13$ & $2.35 \pm 0.13$ & $2.27 \pm 0.21$ & $2.04 \pm 0.1$ & $1.86 \pm 0.14$ \\
\hline Adhesiveness (N s) & $0.04 \pm 0.005$ & $0.04 \pm 0.006$ & $0.03 \pm 0.005$ & $0.03 \pm 0.005$ & $0.05 \pm 0.006$ & $0.03 \pm 0.005$ \\
\hline Cohesiveness (-) & $0.36 \pm 0.01$ & $0.39 \pm 0.01$ & $0.41 \pm 0.01$ & $0.40 \pm 0.01$ & $0.37 \pm 0.01$ & $0.39 \pm 0.01$ \\
\hline Particle size $d_{50}(\mu \mathrm{m})$ & $1935 \pm 28$ & $1944 \pm 27$ & $2008 \pm 25$ & $1968 \pm 19$ & $1936 \pm 20$ & $1961 \pm 16$ \\
\hline Number of particles (-) & $623 \pm 32$ & $555 \pm 27$ & $381 \pm 31$ & $526 \pm 40$ & $561 \pm 37$ & $538 \pm 51$ \\
\hline Saliva ratio $h_{\mathrm{w}}(-)$ & $0.2 \pm 0.02$ & $0.2 \pm 0.01$ & $0.1 \pm 0.02$ & $0.2 \pm 0.01$ & $0.2 \pm 0.02$ & $0.2 \pm 0.04$ \\
\hline
\end{tabular}

Table 3 Path regression coefficients and indirect effects for mediation models. Regression coefficients $a, b$, and $c^{\prime}$ estimate the effect of the predictor on the mediator, the effect of the mediator on the outcome variable, and the direct effect of the predictor on the outcome variable, respectively (see Fig. 1). Indirect effect indicates whether or not a predictor variable had an effect on the outcome variable mediated by the consumption time. When the confidence intervals did not include zero, mediation was considered existent. Lower level confidence interval (LLCI) and upper level confidence interval (ULCI) are indicated

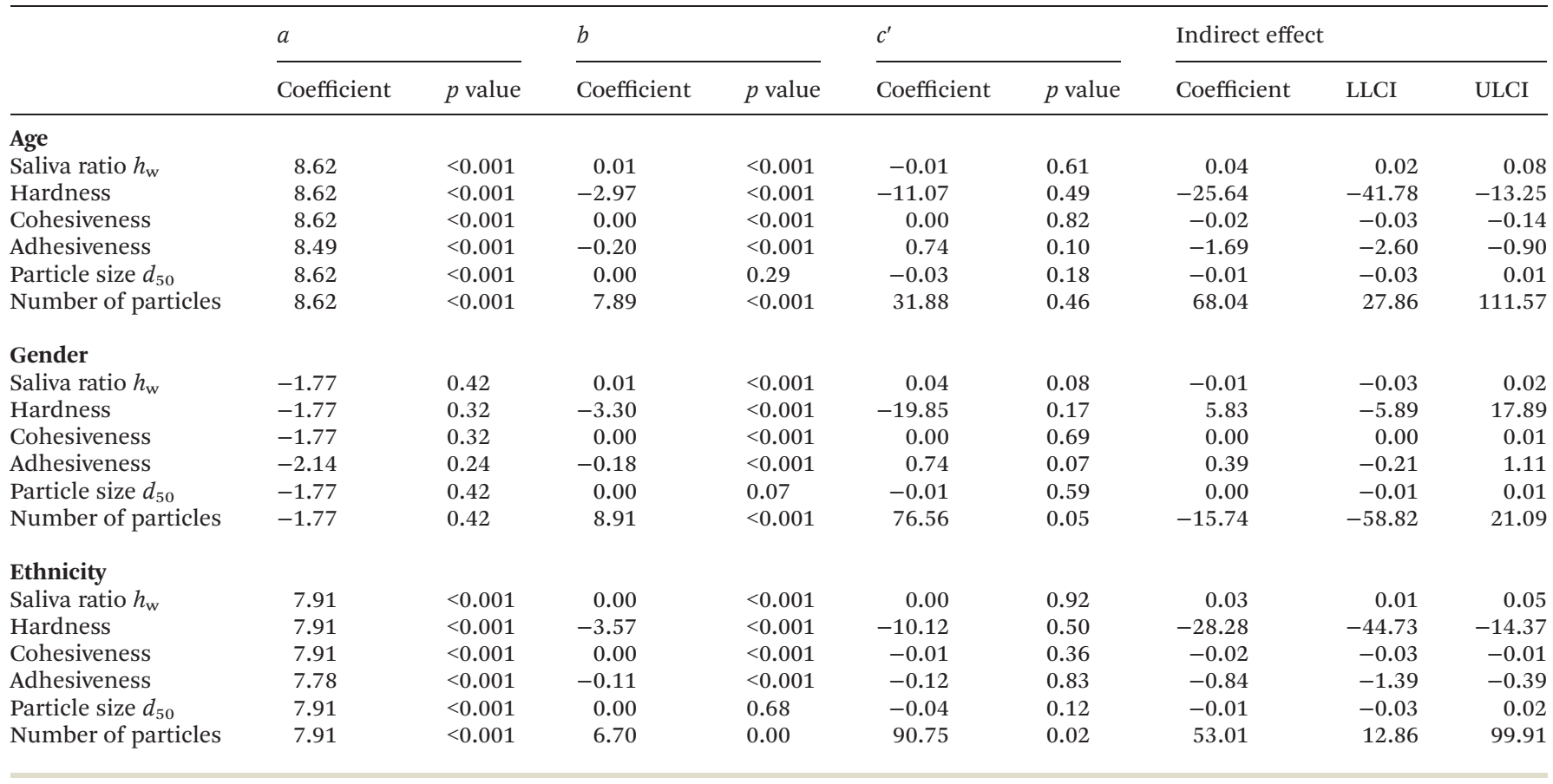

ratio, bolus hardness, cohesiveness, adhesiveness, particle size and number of particles through its effect on consumption time but did not influence bolus particle size. Elderly had a longer consumption time than young adults, and longer consumption time increased saliva ratio and number of particles in the bolus, but decreased hardness, cohesiveness, adhesiveness and particle size. The results of the mediation analysis did not provide evidence that age influenced bolus properties independent of its effect on consumption time. Similarly, gender did not have an indirect effect on bolus properties through consumption time in Dutch consumers.

Ethnicity had an indirect effect on saliva ratio, bolus hardness, adhesiveness, cohesiveness, and number of particles mediated by consumption time. Chinese participants had a longer consumption time than Dutch participants, and participants with a longer consumption time increased saliva ratio and number of particles in the bolus, but decreased hardness, cohesiveness, adhesiveness. The results of the mediation analysis did not provide evidence that ethnicity influenced bolus properties independent of its effect on consumption time.

\subsection{Dynamic texture perception of consumers differing in age, gender and ethnicity}

The TDS curves of the hotdog sausage for the consumer groups differing in age, gender and ethnicity are shown in Fig. 2. Three to four texture attributes were selected as domi- 


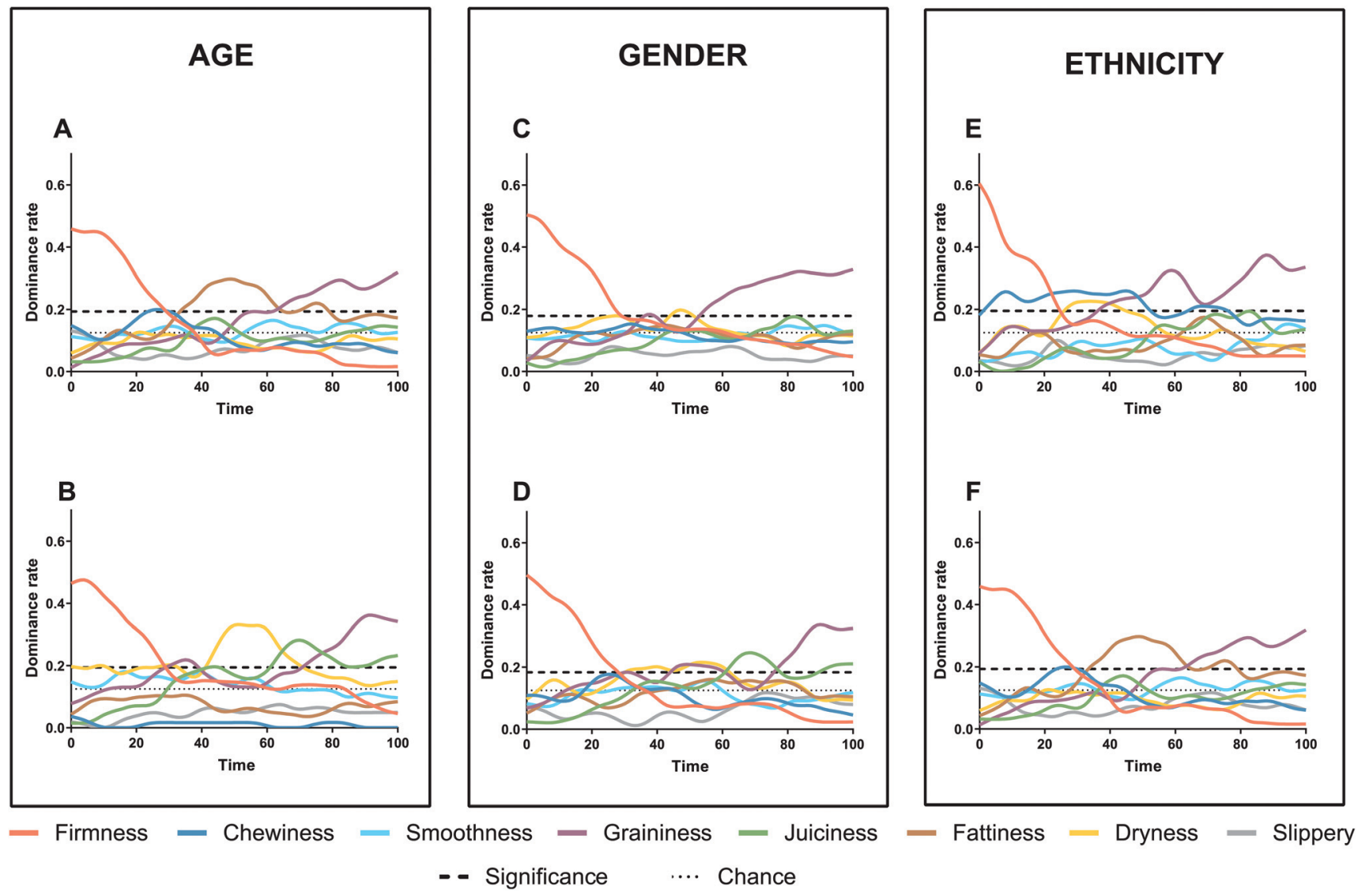

Fig. 2 TDS curves showing texture attribute dominance rates of hotdog sausages in consumers differing in age, gender, and ethnicity. (A and F) show young Dutch; (B) elderly Dutch; (C) females; (D) males; (E) young Chinese.

nant sensations throughout the sensory evaluation by all consumer groups. The dominant texture attributes showed a clear dynamic evolution over consumption time. Fig. 2A-F shows that all consumer groups perceived the texture of the sausage similar during the beginning (first third of normalized consumption time) and end of mastication (last third of normalized consumption time). The dominant texture sensation of the hotdog sausage perceived by all consumer groups at the beginning of mastication was firmness and at the end of mastication it was graininess. Only in the middle of mastication (second third of normalized consumption time) differences in dominant texture sensations were found between consumer groups. It should be noted that dominant texture sensation perceived in the second third of normalized consumption time were significant but displayed low dominance rates for all consumer groups suggesting a low level of panel agreement. For young Dutch adults (Fig. 2A/F) the dominant texture sensation perceived during the second third of consumption time was fattiness. For elderly Dutch the dominant texture sensation perceived during the second third of consumption time was dryness followed by juiciness (Fig. 2B). When comparing the dominant texture sensations between females and males (Fig. 2C and D, respectively), differences are neglectable. Only during the second third of consumption time small differ- ences are perceived but attributes barely reach the significance level. Fig. $2 \mathrm{E}$ and $\mathrm{F}$ show that Chinese consumers selected chewiness and dryness as dominant texture sensation during the second third of consumption time, whereas Dutch consumers selected fattiness.

\subsection{Relationships between dynamic bolus properties and dynamic texture perception}

Multiple Factor Analysis (MFA) was used to explore the relationships between dynamic bolus properties and dynamic texture perception. Fig. 3 displays the first two dimensions of the MFA, both dimensions together explain $59.1 \%$ of the variance in the data. Both groups of data contributed almost equally to Dimension 1, while Dimension 2 was almost exclusively explained by the sensory attributes data. Fig. 3A shows that consumer characteristics (age, gender and ethnicity) are close to the origin of the MFA plot indicating that their contribution to the variance in the data is low. In contrast, the two extreme mastication time points (33 and $100 \%$ mastication times) at which bolus properties and texture perception were determined (TDS data was split into three time periods 33 (T0T33), 66 (T34-T66) and 100 (T67-T100) of standardized consumption time, see section 2.6) are the main contributors to 

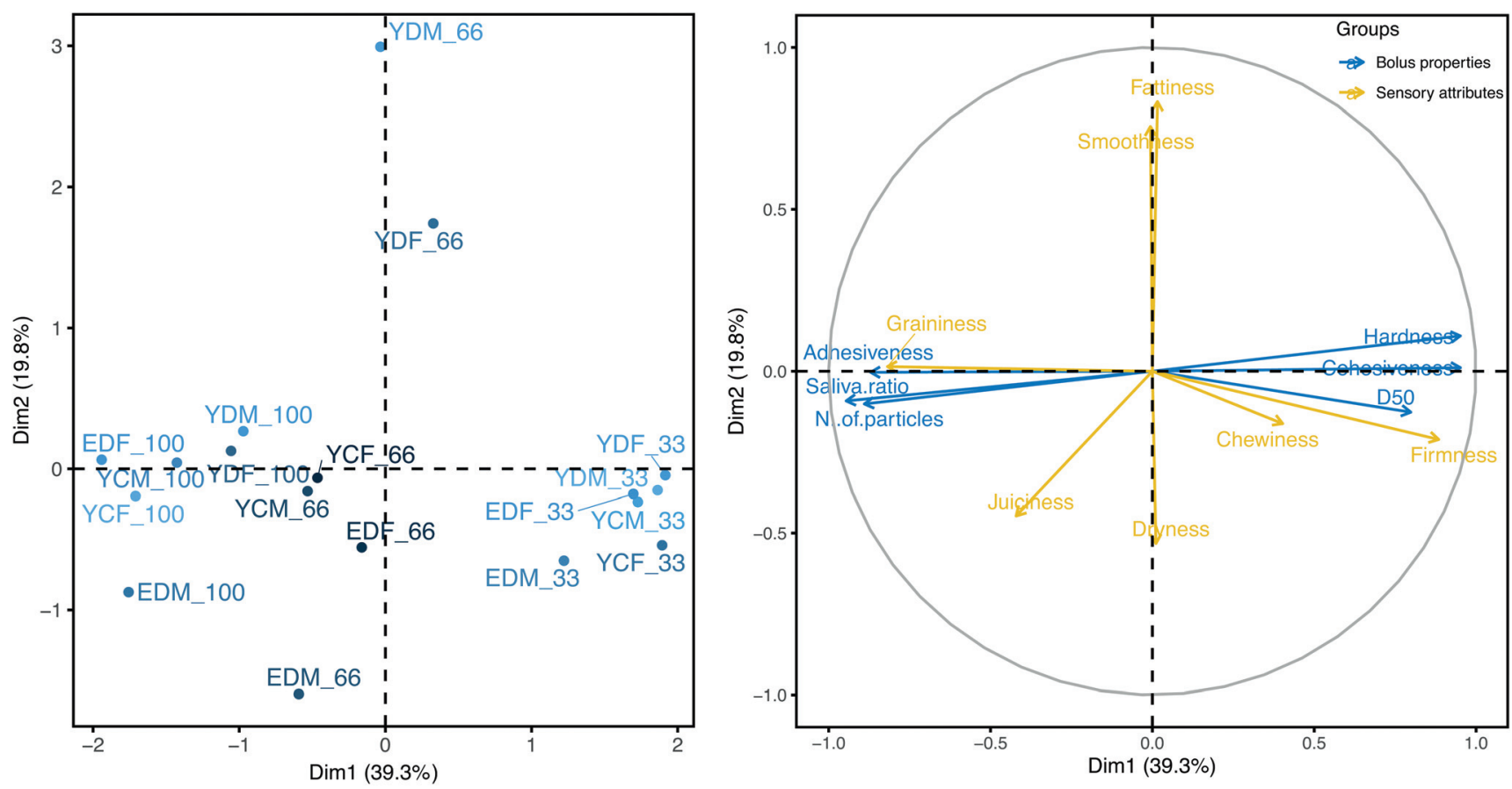

Fig. 3 A. MFA space (first two components) from all consumer groups and mastication times 33, 66 and 100\%. Label codes indicate consumer characteristics and mastication time. First letter refers to age group ( $Y$ for young; $E$ for elderly); second letter to ethnicity (D for Dutch; $C$ for Chinese); third letter to gender ( $M$ for male; $F$ for female); and the number refers to 33, 66 or $100 \%$ of mastication time. B. Correlation circle displaying the relationships between variables of bolus properties (blue) and texture perception determined by TDS (yellow).

the variance in the data. Fig. 3B shows the correlation between dynamic bolus properties and dynamic texture perception.

Overall, MFA shows that at $33 \%$ of mastication time, bolus properties had high values of hardness, cohesiveness and $d_{50}$. These bolus properties were positively correlated among them and with perception of texture attributes firmness and chewiness. At $66 \%$ of mastication time perception of fattiness and smoothness were positively correlated with each other and negatively correlated with dryness (as seen also in Fig. 2). Relative to other attributes, fattiness and smoothness were the attributes mostly reported within young Dutch consumers at that point in time, whereas dryness was mainly reported by elderly Dutch consumers. At $100 \%$ of mastication time, boli were characterized by high amounts of saliva, high adhesiveness and large number of bolus particles. These bolus properties were positively correlated between them and with the perception of graininess and juiciness.

\section{Discussion}

The present study shows that for a fixed bite size of a hotdog sausage, consumers differing in age, gender and ethnicity varied in consumption time. Differences in bolus properties were influenced by age and ethnicity but these effects were mediated by consumption time. This demonstrates that consumption time is the underlying mechanism that explains the differences in bolus properties between consumer groups.
We observed that elderly masticated the hotdog sausages for longer times than young consumers, which is in line with several previous studies showing that among healthy subjects an increment in age is accompanied by an increment in consumption time regardless of the food characteristics. ${ }^{4-6,27}$ This effect has been attributed to a decline of bite ${ }^{28,29}$ and tongue strength with ageing ${ }^{30}$ as well as a reduction in muscle activity and density. ${ }^{31,32}$ Elderly produced a more degraded bolus than young consumers. Thus, consumers differing in age, modify their consumption time and therefore the bolus properties at swallowing point differ considerably in order to reach a bolus with an optimal state for safe and comfortable swallowing. An age related prolongation of consumption time can be an adaptative mechanism not only to overcome the oral decline but also to compensate for a less efficient digestion. ${ }^{33}$ We note that in our study bite size was fixed to $7 \mathrm{~g}$ of hotdog sausage; however, under natural eating conditions, consumers might also adapt their bite size to compensate for a decline in oral capabilities, for example by taking smaller bites leading to shorter consumption times. Our results differ from previous studies by Mioche and colleagues. ${ }^{34}$ They observed that older populations swallow a bolus with lower degree of food structural breakdown. There is a major difference between that study and our study regarding the selection criteria for the dental status of the elderly. In our study only elderly consumers with full denture participated whereas in the study done by Mioche, elderly with at least six pairs of post-canine teeth were included. The worse dental status of the elderly partici- 
pants in Mioche's study might have contributed to the lower degree of structural breakdown of the foods at the moment of swallowing.

Despite the observed changes in bolus properties between young and elderly consumers, dynamic texture perception of the sausage was similar between both groups. Both consumer groups perceived firmness as the first dominant sensation for the first third of consumption time, and with similar dominance rate. The second third of consumption time showed minor differences between young and the elderly in dynamic texture perception. In this period, elderly mainly perceived dryness as dominant sensation, whereas for the young group fattiness was dominant sensation. This period may provide important sensory input to decide if longer mastication is needed in order to reach the bolus lubrication threshold needed for swallowing. Elderly have decreased saliva flow and lower amounts of mucins (salivary proteins that contribute to bolus formation) than younger adults. ${ }^{35,36}$ This means that to reach the same amount of saliva incorporated in a bolus, elderly would need to process the bolus for a longer period of time. This explains why despite significant differences in consumption time, the amount of saliva incorporated into the bolus at the swallowing point was not different between both age groups. During the last third of consumption time graininess was dominant sensation until the swallowing moment. Graininess is an attribute that reflects the increased number of fragments generated during mastication, where particles' size and shape are perceived assessing if they are small enough to be swallow. ${ }^{37}$ The size of bolus particles is another factor that has been suggested to influence the swallowing moment. However, in our study size of bolus particles at the moment of swallowing did not differ between the two age groups.

Gender influenced consumption time but only in Chinese consumers, where Chinese males had shorter consumption time than Chinese females. On the other hand, consumption time differed between Dutch and Chinese but only in females with Chinese females having longer consumption time than Dutch females. Despite the often reported oral physiological differences between males and females and between ethnic groups, ${ }^{28,32,38-41}$ differences in food oral processing behavior related to gender and ethnicity frequently show contrasting results with some studies showing differences in food oral processing behavior ${ }^{5,9,39,42,43}$ and others not. ${ }^{7,44}$ Our study found an interaction effect between gender and ethnicity which may help to explain why conflicting results are found in literature. It is probable that (oral) physiological variations between genders and ethnicities combined with cultural or environmental differences contribute to changes on consumption time. For example, females are frequently taught to slow down their eating rate and chew thoroughly, behavior that might not be encouraged in males as often. ${ }^{8,9,45,46}$ This behavior might be encouraged more often in traditional cultures. Based on the observations made in this study, in order to find more consistent results, investigations about oral behavior should balance the selection of subjects by gender and report or homogenize ethnicity in order to avoid confounding effects.
The comparison between the bolus properties of Dutch and Chinese showed that Chinese expectorated bolus that were softer, less cohesive and contained more particles of slightly smaller size than Dutch whereas the amount of saliva incorporated into the bolus did not differed between both groups. The differences found in bolus texture and bolus particle characteristics might be related to lower mastication efficiency of Chinese compared to Dutch. ${ }^{47}$ Thus, the elongation of consumption time can be a strategy to adapt for lower mastication efficiency.

Dynamic texture perception differed between ethnic groups, only during the second third of consumption time. Dutch consumers perceived the sausage as fatty and Chinese as dry. The difference in texture perception between the ethnicities may be related to physiological factors and cultural expectations. The first factor refers to differences in saliva flow rate between these groups which have been reported previously with Chinese having lower saliva flow rates than Dutch consumers. ${ }^{47}$ Perception of dryness by Chinese, similarly to what was observed in elderly consumers, might contribute to prolongation of consumption time to compensate for low saliva production. In a way that at the swallowing point both ethnicities reach a bolus with the same amount of saliva. The second factor, cultural expectation, may be related to differences in sensory properties between sausages consumed in China and The Netherlands. Many sausages available in China might contain a broad range of fat contents and fat particles might be visible and large. Many sausages available in The Netherlands tend to have lower fat content and typically contain finely ground meat and emulsified fats. ${ }^{48}$ Thus, the expectation of sensory properties of the sausage may be different between the Chinese and Dutch and may drive the attention of both consumers towards different texture attributes.

Gender was the consumer characteristic that had the weakest influence on bolus properties in this study. When gender influenced bolus properties, the effect was always interacting with age or ethnicity. For example, elderly females produced a more adhesive and cohesive bolus than elderly males. Furthermore, among young Dutch consumers, females produced fewer bolus particles than young Dutch males. Similarly to age and ethnicity, gender did not influence the amount of saliva incorporated into the bolus and bolus particle size. It is possible that both saliva incorporation and bolus particle size are key determinants to trigger a swallow. Furthermore, dynamic texture perception did not differ considerably between females and males.

Overall, for all consumer groups, relationships between bolus properties and texture perception were found especially at the beginning and end of consumption time (33 and 100\% mastication time). The first third of consumption time was associated with bolus hardness, cohesiveness, bolus particle size $\left(d_{50}\right)$ and with perception of firmness and chewiness. The bolus properties observed at the beginning of consumption time mainly represent the response of the food structure to mechanical disruption imposed upon chewing. ${ }^{37}$ The positive 
correlation between instrumental bolus hardness and perception of firmness is in agreement with previous studies. ${ }^{49,50}$ Moreover, large particle sizes in foods have been associated with an increment in perception of firmness and chewiness. ${ }^{51}$

The end of consumption time was associated with bolus properties such as saliva ratio, adhesiveness, number of bolus particles and with perception of graininess and juiciness. The increased presence of saliva in the bolus is accompanied by mucins and water, which together can increase the adhesiveness of the bolus contributing to agglomeration of bolus particles. ${ }^{52,53}$ The large number of bolus particles produced during the mastication process can lead to a grainy perception as shown in the present study. To summarize, at the beginning and end of consumption time dynamic texture perception of sausages was similar for all consumer groups and strongly correlated with bolus properties. Minor differences in dynamic texture perception between consumers groups were observed only during the middle stages of mastication with low dominance rates. Consumers varying in age, gender and ethnicity differ in oral processing time of sausages to safely swallow bolus with textural properties optimized for their needs.

\section{Conclusion}

We conclude that consumers differing in age, gender or ethnicity differ in consumption time in order to reach a bolus with optimized textural properties that guarantee a safe and comfortable swallow. The effect of consumer characteristics such as age, gender and ethnicity on bolus properties is mediated by consumption time. While differences in consumption time of sausages leads to considerable differences in bolus properties, it leads only to small differences in dynamic texture perception. Bolus properties and texture perception were mainly correlated at the beginning and end of consumption time. We suggest that the information provided in this study, showing the bolus properties that are needed to enable a safe and comfortable swallow in different consumer groups, helps to optimize foods by tailoring food texture properties towards the needs of specific target groups.

\section{Conflicts of interest}

There are no conflicts of interest to declare.

\section{Acknowledgements}

The project is funded by TiFN, a public-private partnership on precompetitive research in food and nutrition. The public partners are responsible for the study design, data collection and analysis, decision to publish, and preparation of the manuscript. The private partners have contributed to the project through regular discussion. The private partners are Royal Friesland Campina, Fromageries Bel and Unilever. This research was performed with additional funding from the Top
Consortia for Knowledge and Innovation Agri \& Food of the Dutch Ministry of Economic Affairs.

\section{References}

1 M. G. Aguayo-Mendoza, E. C. Ketel, E. van der Linden, C. G. Forde, B. Piqueras-Fiszman and M. Stieger, Oral processing behavior of drinkable, spoonable and chewable foods is primarily determined by rheological and mechanical food properties, Food Qual. Prefer., 2019, 71, 87-95.

2 K. Hiiemae, M. R. Heath, G. Heath, E. Kazazoglu, J. Murray, D. Sapper and K. Hamblett, Natural bites, food consistency and feeding behaviour in man, Arch. Oral Biol., 1996, 41, 175-189.

3 C. Yven, J. Patarin, A. Magnin, H. Labouré, M. Repoux, E. Guichard and G. Feron, Consequences of Individual Chewing Strategies on Bolus Rheological Properties at the Swallowing Threshold, J. Texture Stud., 2012, 43, 309-318.

4 M. Aguayo-Mendoza, M. Santagiuliana, X. Ong, B. Piqueras-Fiszman, E. Scholten and M. Stieger, How addition of peach gel particles to yogurt affects oral behavior, sensory perception and liking of consumers differing in age, Food Res. Int., 2020, 134, 109213.

5 E. C. Ketel, M. G. Aguayo-Mendoza, R. A. de Wijk, C. de Graaf, B. Piqueras-Fiszman and M. Stieger, Age, gender, ethnicity and eating capability influence oral processing behaviour of liquid, semi-solid and solid foods differently, Food Res. Int., 2019, 119, 143-151.

6 K. Kohyama, L. Mioche and J.-F. Martin, Chewing Patterns of Various Texture Foods Studied by Electromyography in Young and Elderly Populations, J. Texture Stud., 2002, 33, 269-283.

7 M.-A. Peyron, O. Blanc, J. P. Lund and A. Woda, Influence of Age on Adaptability of Human Mastication, J. Neurophysiol., 2004, 92, 773-779.

8 S. W. Hill and N. B. McCutcheon, Contributions of obesity, gender, hunger, food preference, and body size to bite size, bite speed, and rate of eating, Appetite, 1984, 5, 73-83.

9 S. Park and W.-S. Shin, Differences in eating behaviors and masticatory performances by gender and obesity status, Physiol. Behav., 2015, 138, 69-74.

10 L. J. Pereira and A. van der Bilt, The influence of oral processing, food perception and social aspects on food consumption: a review, J. Oral Rehabil., 2016, 43, 630-648.

11 M.-A. Peyron, V. Santé-Lhoutellier, O. François and M. Hennequin, Oral declines and mastication deficiencies cause alteration of food bolus properties, Food Funct., 2018, 9, 1112-1122.

12 J. F. Prinz and P. W. Lucas, Swallow thresholds in human mastication, Arch. Oral Biol., 1995, 40, 401-403.

13 J. B. Hutchings and P. J. Lillford, The Perception of Food Texture - the Philosophy of the Breakdown Path, J. Texture Stud., 1988, 19, 103-115.

14 M. Devezeaux de Lavergne, J. A. M. Derks, E. C. Ketel, R. A. de Wijk and M. Stieger, Eating behaviour explains 
differences between individuals in dynamic texture perception of sausages, Food Qual. Prefer., 2015, 41, 189-200.

15 M. Doyennette, M. G. Aguayo-Mendoza, A.-M. Williamson, S. I. F. S. Martins and M. Stieger, Capturing the impact of oral processing behaviour on consumption time and dynamic sensory perception of ice creams differing in hardness, Food Qual. Prefer., 2019, 103721.

16 S. R. Drago, M. Panouillé, A. Saint-Eve, E. Neyraud, G. Feron and I. Souchon, Relationships between saliva and food bolus properties from model dairy products, Food Hydrocolloids, 2011, 25, 659-667.

17 A. Braghieri, N. Piazzolla, F. Galgano, N. Condelli, G. De Rosa and F. Napolitano, Effect of preservative addition on sensory and dynamic profile of Lucanian dry-sausages as assessed by quantitative descriptive analysis and temporal dominance of sensations, Meat Sci., 2016, 122, 68-75.

18 M. M. Farouk, W. K. Hall, M. Harrison and J. E. Swan, Instrumental and Sensory Measurement of Beef Patty and Sausage Texture, J. Muscle Foods, 1999, 10, 17-28.

19 S. Fonseca, M. Gómez, R. Domínguez and J. M. Lorenzo, Physicochemical and sensory properties of Celta dryripened "salchichón" as affected by fat content, Grasas Aceites, 2015, 66, 059.

20 R. C. Jones, E. Dransfield, A. R. Crosland and M. A. Francombe, Sensory characteristics of british sausages: Relationships with composition and mechanical properties, J. Sci. Food Agric., 1989, 48, 63-85.

21 M. Laranjo, A. Gomes, A. C. Agulheiro-Santos, M. E. Potes, M. J. Cabrita, R. Garcia, J. M. Rocha, L. C. Roseiro, M. J. Fernandes, M. H. Fernandes, M. J. Fraqueza and M. Elias, Characterisation of "Catalão" and "Salsichão" Portuguese traditional sausages with salt reduction, Meat Sci., 2016, 116, 34-42.

22 M. Laranjo, A. Gomes, A. C. Agulheiro-Santos, M. E. Potes, M. J. Cabrita, R. Garcia, J. M. Rocha, L. C. Roseiro, M. J. Fernandes, M. J. Fraqueza and M. Elias, Impact of salt reduction on biogenic amines, fatty acids, microbiota, texture and sensory profile in traditional blood dry-cured sausages, Food Chem., 2017, 218, 129-136.

23 K. Paulos, S. Rodrigues, A. F. Oliveira, A. Leite, E. Pereira and A. Teixeira, Sensory Characterization and Consumer Preference Mapping of Fresh Sausages Manufactured with Goat and Sheep Meat, J. Food Sci., 2015, 80, S1568-S1573.

24 R. Solheim, Consumer liking for sausages affected by sensory quality and information on fat content, Appetite, 1992, 19, 285-292.

25 N. Pineau, P. Schlich, S. Cordelle, C. Mathonnière, S. Issanchou, A. Imbert, M. Rogeaux, P. Etiévant and E. Köster, Temporal Dominance of Sensations: Construction of the TDS curves and comparison with timeintensity, Food Qual. Prefer., 2009, 20, 450-455.

26 J. N. Cheong, K. D. Foster, M. P. Morgenstern, J. M. V. Grigor, J. E. Bronlund, S. C. Hutchings and D. I. Hedderley, The Application of Temporal Dominance of Sensations (TDS) for Oral Processing Studies: An Initial Investigation, J. Texture Stud., 2014, 45, 409-419.
27 A. Mishellany-Dutour, J. Renaud, M.-A. Peyron, F. Rimek and A. Woda, Is the goal of mastication reached in young dentates, aged dentates and aged denture wearers?, Br. J. Nutr., 2008, 99, 121-128.

28 M. Bakke, B. Holm, B. L. Jensen, L. Michler and E. Möller, Unilateral, isometric bite force in 8-68-year-old women and men related to occlusal factors, Scand. J. Dent. Res., 1990, 98, 149-158.

29 J. P. Hatch, R. S. A. Shinkai, S. Sakai, J. D. Rugh and E. D. Paunovich, Determinants of masticatory performance in dentate adults, Arch. Oral Biol., 2001, 46, 641-648.

30 H. Koshino, T. Hirai, T. Ishijima and Y. Ikeda, Tongue motor skills and masticatory performance in adult dentates, elderly dentates, and complete denture wearers, J. Prosthet. Dent., 1997, 77, 147-152.

31 J. P. Newton, E. W. Abel, E. M. Robertson and R. Yemm, Changes in human masseter and medial pterygoid muscles with age: a study by computed tomography, Gerodontics, 1987, 3, 151-154.

32 M. Palinkas, M. S. P. Nassar, F. A. Cecílio, S. Siéssere, M. Semprini, J. P. Machado-de-Sousa, J. E. C. Hallak and S. C. H. Regalo, Age and gender influence on maximal bite force and masticatory muscles thickness, Arch. Oral Biol., 2010, 55, 797-802.

33 D. Rémond, D. R. Shahar, D. Gille, P. Pinto, J. Kachal, M.-A. Peyron, C. N. Dos Santos, B. Walther, A. Bordoni, D. Dupont, L. Tomás-Cobos and G. Vergères, Understanding the gastrointestinal tract of the elderly to develop dietary solutions that prevent malnutrition, Oncotarget, 2015, 6, 13858-13898.

34 L. Mioche, P. Bourdiol, S. Monier, J.-F. Martin and D. Cormier, Changes in jaw muscles activity with age: effects on food bolus properties, Physiol. Behav., 2004, 82, 621-627.

35 M. Navazesh, R. A. Mulligan, V. Kipnis, P. A. Denny and P. C. Denny, Comparison of Whole Saliva Flow Rates and Mucin Concentrations in Healthy Caucasian Young and Aged Adults, J. Dent. Res., 1992, 71, 1275-1278.

36 M. Vandenberghe-Descamps, H. Labouré, A. Prot, C. Septier, C. Tournier, G. Feron and C. Sulmont-Rossé, Salivary Flow Decreases in Healthy Elderly People Independently of Dental Status and Drug Intake, J. Texture Stud., 2016, 47, 353-360.

37 S. Fiszman and A. Tarrega, The dynamics of texture perception of hard solid food: A review of the contribution of the temporal dominance of sensations technique, $J$. Texture Stud., 2018, 49, 202-212.

38 H. M. Clark and N. P. Solomon, Age and Sex Differences in Orofacial Strength, Dysphagia, 2012, 27, 2-9.

39 E. C. Ketel, R. A. de Wijk, C. de Graaf and M. Stieger, Relating oral physiology and anatomy of consumers varying in age, gender and ethnicity to food oral processing behavior, Physiol. Behav., 2020, 215, 112766.

40 H. Shiga, Y. Kobayashi, H. Katsuyama, M. Yokoyama and I. Arakawa, Gender difference in masticatory performance in dentate adults, J. Prosthodont. Res., 2012, 56, 166-169. 
41 Z. Zhuang, D. Landsittel, S. Benson, R. Roberge and R. Shaffer, Facial anthropometric differences among gender, ethnicity, and age groups, Ann. Occup. Hyg., 2010, 54, 391-402.

42 T. Nagasawa, X. Yanbin, K. Tsuga and Y. Abe, Sex difference of electromyogram of masticatory muscles and mandibular movement during chewing of food, J. Oral Rehabil., 1997, 24, 605-609.

43 R. E. Youssef, G. S. Throckmorton, E. Ellis and D. P. Sinn, Comparison of habitual masticatory patterns in men and women using a custom computer program, J. Prosthet. Dent., 1997, 78, 179-186.

44 S. Kiliaridis, S. Karlsson and H. Kjellberg, Characteristics of masticatory mandibular movements and velocity in growing individuals and young adults, J. Dent. Res., 1991, 70, 1367-1370.

45 A. Fogel, L. R. Fries, K. McCrickerd, A. T. Goh, M. J. Chan, J. Y. Toh, Y.-S. Chong, K. H. Tan, F. Yap, L. P. Shek, M. J. Meaney, B. F. P. Broekman, Y. S. Lee, K. M. Godfrey, M. F. F. Chong and C. G. Forde, Prospective associations between parental feeding practices and children's oral processing behaviours, Matern. Child. Nutr., 2019, 15, e12635.

46 C. Sook Wah, Gender differences in eating behaviour, IJABM, 2016, 4, 116-121.
47 E. C. Ketel, Understanding variation in food oral processing behavior of consumers differing in age, gender and ethnicity: a physiological perspective, Wageningen University and Research, The Netherlands, 2020, pp. 88-109.

48 C. Y. W. Ang, K. Liu and Y.-W. Huang, Asian Foods: Science and Technology, CRC Press, 1999.

49 R. Di Monaco, S. Cavella and P. Masi, Predicting Sensory Cohesiveness, Hardness and Springiness of Solid Foods from Instrumental Measurements, J. Texture Stud., 2008, 39, 129-149.

50 J.-F. Meullenet, B. G. Lyon, J. A. Carpenter and C. E. Lyon, Relationship Between Sensory and Instrumental Texture Profile Attributes, J. Sens. Stud., 1998, 13, 77-93.

51 M. Santagiuliana, V. Bhaskaran, E. Scholten, B. PiquerasFiszman and M. Stieger, Don't judge new foods by their appearance! How visual and oral sensory cues affect sensory perception and liking of novel, heterogeneous foods, Food Qual. Prefer., 2019, 77, 64-77.

52 P. L. Fuhrmann, M. Aguayo-Mendoza, B. Jansen, M. Stieger and E. Scholten, Characterisation of friction behaviour of intact soft solid foods and food boli, Food Hydrocolloids, 2020, 100, 105441.

53 A. M. Pedersen, A. Bardow, S. B. Jensen and B. Nauntofte, Saliva and gastrointestinal functions of taste, mastication, swallowing and digestion, Oral Dis., 2002, 8, 117-129. 